\title{
Regularity of solutions to a lubrication problem with discountinuous separation data
}

\author{
J. Ignacio Tello
}

\begin{abstract}
We study the regularity of the solution to the Reynolds equation for incompressible and compressible fluids when the gap between the lubricated surfaces, " $h(x, y)$ ", presents a discontinuity in a two-dimensional bounded domain. As in the one-dimensional problem studied by Rayleigh, the solution $P$ does not belong to $C^{1}(\Omega)$ but we obtain that $|\nabla P|$ is bounded, i.e. $P \in W^{1, \infty}(\Omega)$.
\end{abstract}

Keywords: Reynolds equation; Regularity; Elliptic equations

\section{Introduction}

The phenomenon of Lubrication, already known by the very early civilizations, has been the object of much interest and research; particularly in the field of engineering where it has helped to solve problems related to friction in rollers, journal bearings, gears, thrust pads and other mechanisms.

One of the earlier mathematical models was presented by Reynolds in [11] starting a very rich literature on this subject. For the purposes of this paper we pay attention to Reynolds equation, where the distance $h$ between the lubricated surfaces is small, assuming that one of the surfaces is given by $z=0$ and that it moves with a given velocity $(U, 0,0)$ (i.e. parallel to the $x$-axis). The pressure of the lubricant is denoted by $P$. The incompressible Reynolds equation has the expression

$$
\begin{cases}-\operatorname{div}\left(h^{3} \nabla P\right)=-6 \mu U \frac{\partial}{\partial x} h & \text { in } \Omega \\ P-P_{0}=0 & \text { on } \partial \Omega\end{cases}
$$


where $\mu$ is the viscosity of the fluid and $P_{0}$ the pressure at the boundary. The compressible equation is given by

$$
\begin{cases}-\operatorname{div}\left(h^{3} P \nabla P\right)=-6 \mu U \frac{\partial}{\partial x}(h P) & \text { in } \Omega, \\ P-P_{0}=0 & \text { on } \partial \Omega .\end{cases}
$$

The classical Reynolds equation (1.2) was later modified for many different purposes. Some of these modifications take into account the slip flow and, therefore, the diffusion coefficient $h^{3} P$ is replaced by $\alpha h^{2}+\beta h^{3} P$ (see [3]). Here, we assume the mean free path of the molecules $\lambda$ is small in comparison to the film thickness $h$ (i.e. $\lambda / h<10^{-2}$ ) and so we shall work with the classical Reynolds equation (1.2).

In [10] Rayleigh considered the one-dimensional problem. He optimized the load carrying capacity obtaining the optimal form of " $h$ " given by

$$
h(x)= \begin{cases}h_{0} & \text { if } x \in\left(0, L_{1}\right), \\ h_{1} & \text { if } x \in\left(L_{1}, L\right)\end{cases}
$$

whose solution $P$ is given by

$$
P= \begin{cases}P_{0}+A x & \text { if } x \in\left(0, L_{1}\right), \\ P_{0}+\frac{A L_{1}}{L-L_{1}}(L-x) & \text { if } x \in\left(L_{1}, L\right),\end{cases}
$$

where $A=A\left(\mu, U, h_{0}, h_{1}\right)$. This explicit solution to the one-dimensional problem belongs to the Sobolev space $W^{1, \infty}(0, L)$, i.e. $\left|P_{x}\right|<C$ (see [4]). Nevertheless, notice that $P \notin$ $C^{1}(0, L)$.

During the last century many phenomena have been studied in lubrication theory, such as for instances cavitation or deformation of the surfaces. Many authors have coupled the Reynolds equation to other equations in order to describe the new phenomena, see $[1,3,4,8,12]$, etc. Other authors have studied the Reynolds Equation from a theoretical point of view (see e.g. $[5,6]$ ).

Problems where lubricated surfaces present discontinuities of type (1.3) frequently appear in different engineering applications, such as in "feedbox", "shaft-bearing" systems, "magnetic head recording" or in "hard or floppy disc drives" (see [2] and also [9]).

In this paper we study the regularity of the solution to $(1.1)$ and $(1.2)$ where $\Omega=$ $(0, L) \times(0, B)$ and $h$ is given by the discontinuous function

$$
h(x, y)= \begin{cases}h_{0} & \text { if } x \in\left(0, \frac{L}{2}\right) \\ h_{1} & \text { if } x \in\left(\frac{L}{2}, L\right) .\end{cases}
$$

Since $h$ presents a discontinuity the source term $\partial h / \partial x \approx \delta_{x=L_{1}}$ and we cannot expect that the solution $P$ belongs to $C^{2}(\Omega)$. For this reason we need to introduce the standard notion of week solution, given in Definitions 2.1 and 3.1.

The regularity $C^{0, \alpha}(\Omega)$ for all $\alpha \in[0,1)$ is a direct consequence of the regularity theory (see e.g. [9, Theorem 9.2]). The $W^{1, p}(\Omega)$ regularity is a more delicate question due to the lack of continuity of $h$. The results of Section 2 were advanced in [7]. 
Theorem 1.1. Let $P$ be the unique weak solution to $(1.1)$. Then $u:=P-P_{0} \in W_{0}^{1, \infty}(\Omega)$.

Theorem 1.2. Let $P$ be a weak solution of (1.2). Then $P \in W^{1, \infty}(\Omega)$.

The proofs of the above theorems are presented in Sections 2 and 3, respectively. We consider an auxiliary problem in order to construct a test function which helps us to obtain the needed estimates in the regularized problems (see (2.3) and (3.2)). Once obtained the needed estimates, we take limits in the weak formulation to obtain the desired results.

In this paper we assume

$$
h_{0}<h_{1} \text { and } U<0 \text {, }
$$

$n$ denotes the outward unit normal to $\partial \Omega$ and $\mathrm{d} \sigma$ denotes $\mathrm{d} x \mathrm{~d} y$.

\section{Incompressible case}

Since $h \in L^{\infty}(\Omega)$ and $-6 \mu U \partial h / \partial x \in H^{-1}(\Omega)$, we have

$$
\left\langle-6 \mu U \frac{\partial h}{\partial x}, \phi\right\rangle_{H^{-1}(\Omega) \times H_{0}^{1}(\Omega)}=6 \mu U \int_{\Omega} h \frac{\partial \phi}{\partial x} \mathrm{~d} \sigma, \quad \phi \in H_{0}^{1}(\Omega) .
$$

Definition 2.1. We say that $P$ is a weak solution of (1.1) if $P=u+P_{0}, u \in H_{0}^{1}(\Omega)$ and $u$ satisfies

$$
\int_{\Omega} h^{3} \nabla u \cdot \nabla \phi \mathrm{d} \sigma=\int_{\Omega} 6 \mu U h \frac{\partial \phi}{\partial x} \mathrm{~d} \sigma \quad \forall \phi \in H_{0}^{1}(\Omega)
$$

The existence of a unique weak solution is a direct application of Lax-Milgram theorem. The proof of Theorem 1.1 is structured in several steps. In the first step we approximate $h$ by some continuous functions $h_{\varepsilon}$,

$$
h_{\varepsilon}(x, y)= \begin{cases}h_{0} & \text { if } 0 \leqslant x \leqslant \frac{L}{2} \\ \frac{1}{\varepsilon}\left(x-\frac{L}{2}+\varepsilon h_{0}\right) & \text { if } \frac{L}{2} \leqslant x \leqslant \frac{L}{2}+\varepsilon\left(h_{1}-h_{0}\right), \\ h_{1} & \text { if } \frac{L}{2}+\varepsilon\left(h_{1}-h_{0}\right) \leqslant x \leqslant L\end{cases}
$$

and consider the approximated problem

$$
\begin{cases}-\operatorname{div}\left(h_{\varepsilon}^{3} \nabla u_{\varepsilon}\right)=-6 \mu U \frac{\partial}{\partial x}\left(h_{\varepsilon}\right) & \text { in } \Omega \\ u_{\varepsilon}=0 & \text { on } \partial \Omega .\end{cases}
$$

We have the following proposition.

Proposition 2.1. The solution $u_{\varepsilon}$ to (2.3) belongs to $W_{0}^{1, q}(\Omega)$ for any $1 \leqslant q<\infty$.

According to Troianiello [13, Theorems 3.7 and 3.14] the proof of the above result reduces to prove that the outer normal derivative $\partial u_{\varepsilon} / \partial n$ is a bounded function. It is deduced from the following lemma. 
Lemma 2.1. $\partial u_{\varepsilon} / \partial n \in L^{\infty}(\partial \Omega)$.

Proof. Define the operator $L_{\varepsilon}(\cdot)=-\operatorname{div}\left(h_{\varepsilon}^{3} \nabla(\cdot)\right)$. Then we have

$$
L_{\varepsilon}\left(u_{\varepsilon}\right)=-6 \mu U \frac{\partial}{\partial x}\left(h_{\varepsilon}\right)= \begin{cases}0, & \text { if } 0 \leqslant x \leqslant \frac{L}{2}, \\ -\frac{6 \mu U}{\varepsilon} & \text { if } \frac{L}{2} \leqslant x \leqslant \frac{L}{2}+\varepsilon\left(h_{1}-h_{0}\right), \\ 0 & \text { if } \frac{L}{2}+\varepsilon\left(h_{1}-h_{0}\right) \leqslant x \leqslant L .\end{cases}
$$

Let $\bar{u}_{\varepsilon}(x, y)=C x(x-L) y(y-B)$ with $C=48 \mu|U| / h_{0}^{3} L^{2} \varepsilon$. A routine computation shows that

$$
\begin{aligned}
& L_{\varepsilon}\left(\bar{u}_{\varepsilon}\right) \\
& \quad= \begin{cases}-2 C h_{0}^{3} y(y-B)-2 C h_{0}^{3} x(x-L) & \text { on } 0 \leqslant x \leqslant \frac{L}{2}, \\
-2 C h_{1}^{3} y(y-B)-2 h_{1}^{3} x(x-L) & \text { on } \frac{L}{2}+\varepsilon\left(h_{1}-h_{0}\right) \leqslant x \leqslant L, \\
-C y(y-B)\left[2 h_{\varepsilon}^{3}+\frac{3}{\varepsilon}(2 x-L) h_{\varepsilon}^{2}\right]-C h_{\varepsilon}^{3} x(x-L) & \text { otherwise. }\end{cases}
\end{aligned}
$$

Since $L_{\varepsilon}\left(\bar{u}_{\varepsilon}\right)>L_{\varepsilon}\left(u_{\varepsilon}\right) \geqslant L_{\varepsilon}(0)=0$ in $\Omega$, and $\bar{u}_{\varepsilon}=u_{\varepsilon}=0$ on $\partial \Omega$, we get that $\bar{u}_{\varepsilon}$ is a supersolution and 0 is a subsolution to (2.1). Thus, by the comparison principle and the uniqueness of solutions, we obtain $\bar{u}_{\varepsilon} \geqslant u_{\varepsilon} \geqslant 0$ in $\Omega$. In consequence

$$
\frac{\partial \bar{u}_{\varepsilon}}{\partial n} \leqslant \frac{\partial u_{\varepsilon}}{\partial n} \leqslant 0
$$

which implies that

$$
-C\left(B^{2}+L^{2}\right) \leqslant \frac{\partial u_{\varepsilon}}{\partial n} \leqslant 0 \quad \text { and so } \frac{\partial u_{\varepsilon}}{\partial n} \in L^{\infty}(\partial \Omega) .
$$

In a second step, given $q>1$, we construct a test function $w_{\varepsilon}$ as solution to the auxiliary problem

$$
\begin{cases}-\operatorname{div}\left(h_{\varepsilon}^{3} \nabla w_{\varepsilon}\right)=-\operatorname{div}\left(\left|\nabla u_{\varepsilon}\right|^{q-2} \nabla u_{\varepsilon}\right) & \text { in } \Omega \\ h_{\varepsilon}^{3} \frac{\partial w_{\varepsilon}}{\partial \vec{n}}+a_{\varepsilon}(x, y) w_{\varepsilon}=\left|\nabla u_{\varepsilon}\right|^{q-2} \frac{\partial u_{\varepsilon}}{\partial n} & \text { on } \partial \Omega,\end{cases}
$$

where

$$
\begin{aligned}
& a_{\varepsilon}(x, y):=G_{\varepsilon}^{-1}\left(h_{\varepsilon}^{3} \frac{\partial u_{\varepsilon}}{\partial n}-h_{\varepsilon}^{3} \frac{\partial G_{\varepsilon}}{\partial n}\right), \\
& G_{\varepsilon}(x):=\int_{0}^{x} \frac{6 \mu U}{h_{\varepsilon}^{2}(s)} \mathrm{d} s-k_{0},
\end{aligned}
$$

and

$$
k_{0}:=-\frac{\left(\left\|\partial u_{\varepsilon} / \partial n\right\|_{L^{\infty}(\partial \Omega)}^{q}+|U| h_{1}\right) C^{2}(\Omega) h_{0}^{3}}{6 \mu} .
$$

In order to solve (2.4) we introduce the Hilbert space

$$
V(\Omega):=\left\{\phi \in H^{1}(\Omega) \text { such that } \int_{\partial \Omega} a_{\varepsilon} \phi \mathrm{d} \sigma_{2}=0\right\}
$$

associated to the inner product $\langle\phi, \psi\rangle_{V}:=\int_{\Omega} \nabla \phi \cdot \nabla \psi \mathrm{d} \sigma+\left(\int_{\Omega} \phi \mathrm{d} \sigma\right)\left(\int_{\Omega} \psi \mathrm{d} \sigma\right)$. 
Proposition 2.2. Problem (2.4) has a unique weak solution $w_{\varepsilon} \in V(\Omega)$.

For the proof we need a previous result.

Lemma 2.2. $\int_{\partial \Omega} a_{\varepsilon} \mathrm{d} \sigma_{2}<0$ for any $\varepsilon>0$.

Proof. Integrating in (2.3) it results

$$
\int_{\partial \Omega} h_{\varepsilon}^{3} \frac{\partial u_{\varepsilon}}{\partial n}-h_{\varepsilon}^{3} \frac{\partial G_{\varepsilon}}{\partial n} \mathrm{~d} \sigma_{2}=0
$$

By construction of $a_{\varepsilon}$ we have

$$
\int_{\partial \Omega} a_{\varepsilon} \mathrm{d} \sigma_{2}=\int_{\partial \Omega}\left(G_{\varepsilon}^{-1}-G^{-1}(0)\right)\left(h_{\varepsilon}^{3} \frac{\partial u_{\varepsilon}}{\partial n}-h_{\varepsilon}^{3} \frac{\partial G_{\varepsilon}}{\partial n}\right) \mathrm{d} \sigma_{2} .
$$

Since (by Lemma 2.1)

$$
\frac{\partial u_{\varepsilon}}{\partial n} \leqslant 0 \text { on } \partial \Omega, \quad \frac{\partial G_{\varepsilon}}{\partial n}=0 \text { on } y(B-y)=0, \quad \frac{\partial G_{\varepsilon}}{\partial n}>0 \quad \text { on } x=L
$$

and $G_{\varepsilon}^{-1}-G^{-1}(0)>0$ we obtain the desired result.

We point out that any function in $H^{1}(\Omega)$ can be expressed as the sum of a function in $V(\Omega)$ plus a constant.

Proof of Proposition 2.2. We define the bilinear form $A_{\varepsilon}: V(\Omega) \times V(\Omega) \rightarrow \mathbb{R}$ by

$$
A_{\varepsilon}(\phi, \psi):=\int_{\Omega} h_{\varepsilon}^{3} \nabla \psi \cdot \nabla \phi \mathrm{d} \sigma+\int_{\partial \Omega} a_{\varepsilon} \psi \phi \mathrm{d} \sigma_{2} .
$$

Since

$$
\begin{aligned}
\int_{\partial \Omega} a_{\varepsilon} \psi \phi \mathrm{d} \sigma_{2}= & \int_{\partial \Omega} a_{\delta}\left(\psi-\frac{1}{|\Omega|} \int_{\Omega} \psi \mathrm{d} \sigma\right)\left(\phi-\frac{1}{|\Omega|} \int_{\Omega} \phi \mathrm{d} \sigma\right) \mathrm{d} \sigma_{2} \\
& -\frac{1}{|\Omega|^{2}} \int_{\partial \Omega} a_{\varepsilon} \mathrm{d} \sigma_{2} \int_{\Omega} \psi \mathrm{d} \sigma \int_{\Omega} \phi \mathrm{d} \sigma
\end{aligned}
$$

and $\left\|\psi-(1 /|\Omega|) \int_{\Omega} \psi\right\|_{L^{2}(\partial \Omega)} \leqslant C(\Omega)\|\nabla \psi\|_{\left(L^{2}(\Omega)\right)^{2}}$ we obtain the continuity of $A_{\varepsilon}$. Coerciveness is a consequence of the fact that $\int_{\partial \Omega} a_{\varepsilon}<0$. Since $A_{\varepsilon}$ is a continuous, coercive and bilinear form, by the Lax-Milgram Theorem, there exists a unique $w_{\varepsilon} \in V(\Omega)$ weak solution to (2.3).

Lemma 2.3. There exists a positive constant $K\left(\Omega, h_{0}, U, \mu\right)$ such that

$$
\left\|u_{\varepsilon}\right\|_{W_{0}^{1, q}(\Omega)} \leqslant K\left(\Omega, h_{0}, U, \mu\right) \quad \forall 1<q<\infty .
$$

Proof. We take $w_{\varepsilon}$ as test function in (2.3) and we get

$$
\begin{aligned}
\int_{\Omega} h_{\varepsilon}^{3} \nabla u_{\varepsilon} \cdot \nabla w_{\varepsilon} \mathrm{d} \sigma= & \int_{\Omega} \nabla w_{\varepsilon} \cdot\left(6 \mu U h_{\varepsilon}, 0\right) \mathrm{d} \sigma \\
& +\int_{\partial \Omega} h_{\varepsilon}^{3}\left(\frac{\partial u_{\varepsilon}}{\partial n}-\left\langle\left(6 \mu U h_{\varepsilon}, 0\right), n\right\rangle\right) w_{\varepsilon} \mathrm{d} \sigma_{2} .
\end{aligned}
$$


Taking $u_{\varepsilon}$ as test function in (2.4) we obtain

$$
\int_{\Omega} h_{\varepsilon}^{3} \nabla u_{\varepsilon} \cdot \nabla w_{\varepsilon} \mathrm{d} \sigma=\int_{\Omega}\left|\nabla u_{\varepsilon}\right|^{q} \mathrm{~d} \sigma
$$

Therefore,

$$
\begin{aligned}
\int_{\Omega}\left|\nabla u_{\varepsilon}\right|^{q} \mathrm{~d} \sigma= & \int_{\Omega} \nabla w_{\varepsilon} \cdot\left(6 \mu U h_{\varepsilon}, 0\right) \mathrm{d} \sigma \\
& +\int_{\partial \Omega}\left(h_{\varepsilon}^{3} \frac{\partial u_{\varepsilon}}{\partial n}-\left\langle\left(6 \mu U h_{\varepsilon}, 0\right), n\right\rangle\right) w_{\varepsilon} \mathrm{d} \sigma_{2} .
\end{aligned}
$$

By construction of $G_{\varepsilon}$ we know that $h_{\varepsilon}^{3} \nabla G_{\varepsilon}=\left(6 \mu U h_{\varepsilon}, 0\right)$. Thus

$$
\int_{\Omega} \nabla w_{\varepsilon} \cdot\left(6 \mu U h_{\varepsilon}, 0\right) \mathrm{d} \sigma=\int_{\Omega} h_{\varepsilon}^{3} \nabla G_{\varepsilon} \cdot \nabla w_{\varepsilon} \mathrm{d} \sigma
$$

Taking $G_{\varepsilon}$ as test function in (2.4) we obtain

$$
\begin{aligned}
\int_{\Omega} h_{\varepsilon}^{3} \nabla G_{\varepsilon} \cdot \nabla w_{\varepsilon} \mathrm{d} \sigma= & \int_{\Omega}\left|\nabla u_{\varepsilon}\right|^{q-2} \nabla u_{\varepsilon} \cdot \nabla G_{\varepsilon} \mathrm{d} \sigma \\
& -\int_{\partial \Omega} G_{\varepsilon}\left|\nabla u_{\varepsilon}\right|^{q-2} \frac{\partial u_{\varepsilon}}{\partial n} \mathrm{~d} \sigma_{2}+\left\langle G_{\varepsilon}, h_{\varepsilon}^{3} \frac{\partial w_{\varepsilon}}{\partial n}\right\rangle_{H^{1 / 2, H-1 / 2}} .
\end{aligned}
$$

From (2.6), (2.7) and the above expression we get

$$
\begin{aligned}
\int_{\Omega}\left|\nabla u_{\varepsilon}\right|^{q} \mathrm{~d} \sigma= & \int_{\Omega}\left|\nabla u_{\varepsilon}\right|^{q-2} \nabla u_{\varepsilon} \cdot \nabla G_{\varepsilon} \mathrm{d} \sigma-\int_{\partial \Omega} G_{\varepsilon}\left|\nabla u_{\varepsilon}\right|^{q-2} \frac{\partial u_{\varepsilon}}{\partial n} \mathrm{~d} \sigma_{2} \\
& +\left\langle G_{\varepsilon}, h_{\varepsilon}^{3} \frac{\partial w_{\varepsilon}}{\partial n}\right\rangle_{H^{1 / 2}, H^{-1 / 2}}+\int_{\partial \Omega}\left(h_{\varepsilon}^{3} \frac{\partial u_{\varepsilon}}{\partial n}-\left\langle\left(6 \mu U h_{\varepsilon}, 0\right), n\right\rangle\right) w_{\varepsilon} \mathrm{d} \sigma_{2} .
\end{aligned}
$$

Using the definition of $w_{\varepsilon}$ we arrive at

$$
\int_{\Omega}\left|\nabla u_{\varepsilon}\right|^{q} \mathrm{~d} \sigma=\int_{\Omega}\left|\nabla u_{\varepsilon}\right|^{q-2} \nabla u_{\varepsilon} \cdot \nabla G_{\varepsilon} \mathrm{d} \sigma
$$

which implies

$$
\int_{\Omega}\left|\nabla u_{\varepsilon}\right|^{q} \mathrm{~d} \sigma \leqslant\left\|\nabla G_{\varepsilon}\right\|_{\left\{L^{\infty}(\Omega)\right)^{2}} \int_{\Omega}\left|\nabla u_{\varepsilon}\right|^{q-1} \mathrm{~d} \sigma .
$$

In consequence

$$
\left\|\nabla u_{\varepsilon}\right\|_{\left(L^{q}\right)^{2}}^{q} \leqslant \frac{6 \mu U}{h_{0}^{2}}\left\|\nabla u_{\varepsilon}\right\|_{\left(L^{g-1}(\Omega)\right)^{2}}^{q-1} \leqslant C(\Omega) \frac{6 \mu U}{h_{0}^{2}}\left\|\nabla u_{\varepsilon}\right\|_{\left(L^{q}(\Omega)\right)^{2}}^{q-1},
$$

i.e. $\left\|\nabla u_{\varepsilon}\right\|_{\left(L^{q}\right)^{2}} \leqslant C(\Omega) 6 \mu U / h_{0}^{2}$, and so we conclude that $\left\|u_{\varepsilon}\right\|_{W_{0}^{1, q}} \leqslant C(\Omega) 6 \mu U / h_{0}^{2}$ for any $q \in(1, \infty)$.

End of the proof of Theorem 1. Since $\left\|u_{\varepsilon}\right\|_{W_{0}^{1, q}(\Omega)} \leqslant C$, then there exists a subsequence $u_{\varepsilon i}$ which converges weakly in $W_{0}^{1, q}(\Omega)$ to $v$. Taking limits in the weak formulation of (2.3), we get that $v$ is a solution to (1.1). From the uniqueness of solution we deduce that $u=v$ and then $u \in W_{0}^{1, q}(\Omega)$ for any $q<\infty$. Moreover $\|u\|_{W_{0}^{1, q}(\Omega)} \leqslant C\left(\Omega, h_{0}, \mu, U\right)$ 
which is independent of $q$. Since $\|u\|_{W_{0}^{1, \infty}}=\lim _{q \rightarrow \infty}\|u\|_{W_{0}^{1, q}}$ taking limits as $q \rightarrow \infty$ we conclude the proof.

Lemma 2.4. The solution $P$ to problem (2.1) is such that $P \notin C^{1}(\Omega)$.

Proof. Since $-\Delta P=0$ outside of $x=L / 2$, and $P \geqslant P_{0}, P$ takes his maximum at some point $\left(x_{0}, y_{0}\right)$, where $x_{0}=L / 2$ and $0<y_{0}<B$. Consider the equation

$$
\begin{cases}-\Delta P_{1}=0 & \text { in } \Omega_{1}, \\ P_{1}=P & \text { on } \partial \Omega_{1},\end{cases}
$$

where $\Omega_{1}$ is an open subset of $\Omega$ with regular boundary such that $\Omega_{1} \subset \Omega \cap\{0<$ $x<L / 2\}$ and $\left(L / 2, y_{0}\right),\left(0, y_{0}\right)$ belonging to $\partial \Omega_{1}$. By the strong maximum principle we obtain

$$
\frac{\partial}{\partial x} P_{1}>0
$$

In the same way, we consider $P_{2}, \Omega_{2} \subset \Omega \cap\{L / 2<x<L\}$ and $\left(L / 2, y_{0}\right),\left(L, y_{0}\right)$ belonging to $\partial \Omega_{2}$ and we obtain

$$
\frac{\partial}{\partial x} P_{2}<0
$$

By the uniqueness of the solution it results $P_{1}=P$ in $\Omega_{1}$ and $P_{2}=P$ in $\Omega_{2}$ and by (2.8) and (2.9) we get

$$
\lim _{(x, y) \rightarrow\left(L / 2, y_{0}\right), x<L / 2} \frac{\partial}{\partial x} P>0>\lim _{(x, y) \rightarrow\left(L / 2, y_{0}\right), x>L / 2} \frac{\partial}{\partial x} P,
$$

which proves the lemma.

\section{Compressible case}

In this section we study the compressible case modeled by the compressible Reynolds equation (1.2), where $h$ is given by (1.3). As in Section 2 we introduce the standard notion of weak solution.

Definition 3.1. We say $P \geqslant 0$ is a weak solution to (1.2) if $P-P_{0} \in H_{0}^{1}(\Omega) \cap L^{\infty}(\Omega)$ satisfies

$$
\int_{\Omega} h^{3} P \nabla P \cdot \nabla \psi \mathrm{d} \sigma=\int_{\Omega} 6 \mu U h P \frac{\partial \psi}{\partial x} \mathrm{~d} \sigma \quad \forall \psi \in H_{0}^{1}(\Omega) .
$$

The proof of Theorem 1.2 consists of several steps. First we introduce the unknown function $u=\frac{1}{2} P^{2}$ which satisfies

$$
\begin{cases}-\operatorname{div}\left(h^{3} \nabla u\right)=-6 \sqrt{2} U \mu \frac{\partial}{\partial x}(h \sqrt{u}) & \text { in } \Omega, \\ u-\frac{1}{2} P_{0}^{2}=0 & \text { on } \partial \Omega .\end{cases}
$$


The existence of weak solution can be proved in several ways: using a fixed point theorem (see [5]) or as limit of the solutions of some regular approximated problems.

Let us consider a continuous approximation of $h$ (given by $(2.2)$ ) and the approximated problem

$$
\begin{cases}-\operatorname{div}\left(h_{\varepsilon}^{3} \nabla u_{\varepsilon}\right)=-6 \sqrt{2} U \mu \frac{\partial}{\partial x}\left(h_{\varepsilon} \sqrt{u_{\varepsilon}}\right) & \text { in } \Omega, \\ u_{\varepsilon}=\frac{1}{2} P_{0}^{2} & \text { on } \partial \Omega .\end{cases}
$$

Then

Lemma 3.1. There exists a weak solution to (3.2). Furthermore, the weak solution $u_{s}$ belongs to $W^{1, q}(\Omega)$ for any $1 \leqslant q<\infty$.

Proof. Let us consider the operator

$$
L_{\varepsilon}(\cdot)=-\operatorname{div}\left(h_{\varepsilon}^{3} \nabla(\cdot)\right)+6 \sqrt{2} \mu U \frac{\partial}{\partial x}\left(h_{\varepsilon} \sqrt{(\cdot)}\right) .
$$

Let $u_{0}=\frac{1}{2} P_{0}^{2}$, then by (1.4) we get $L_{\varepsilon}\left(u_{0}\right) \leqslant 0$. Let us consider $\bar{u}_{\varepsilon}$ defined by $\bar{u}_{\varepsilon}(x, y)=$ $C x(x-L) y(y-B)+P_{0}^{2}$, where $C=\left(12 \mu B U_{0} / \varepsilon h_{0}^{3} L\right)^{2}$. Then we have

$$
\begin{cases}L_{\varepsilon}\left(\bar{u}_{\varepsilon}\right)>L_{\varepsilon}\left(u_{\varepsilon}\right) \geqslant L_{\varepsilon}\left(u_{0}\right) & \text { in } \Omega, \\ \bar{u}_{\varepsilon}=u_{\varepsilon}=u_{0} & \text { on } \partial \Omega\end{cases}
$$

and by comparison we obtain the existence of solution $u_{\varepsilon}$ satisfying

$$
\bar{u}_{\varepsilon} \geqslant u_{\varepsilon} \geqslant P_{0}^{2} \quad \text { in } \Omega \text {. }
$$

Then

$$
\frac{\partial \bar{u}_{\varepsilon}}{\partial n} \leqslant \frac{\partial u_{\varepsilon}}{\partial n} \leqslant 0
$$

and we get

$$
-C\left(B^{2}+L^{2}\right) \leqslant \frac{\partial u_{\varepsilon}}{\partial n} \leqslant 0
$$

The second step of the proof of Theorem 1.2 is to prove the regularity of $P_{\varepsilon}=\sqrt{2 u_{\varepsilon}}$, where $P_{\varepsilon}$ is the solution to

$$
\begin{cases}-\operatorname{div}\left(h_{\varepsilon}^{3} P_{\varepsilon} \nabla P_{\varepsilon}\right)=-6 \mu U \frac{\partial}{\partial x}\left(h_{\varepsilon} P_{\varepsilon}\right) & \text { in } \Omega \\ P_{\varepsilon}=P_{0} & \text { on } \partial \Omega\end{cases}
$$

Proposition 3.1. The solution to (3.3) satisfies

$$
\left\|P_{\varepsilon}\right\|_{W^{1, q}(\Omega)} \leqslant K
$$

for any $1 \leqslant q<\infty$ and $K=K\left(\Omega, h_{0}, U_{0}, \mu, P_{0}\right)$, i.e. independent of $q$ and $\varepsilon$. 
In order to prove Proposition 3.1, given $q>1$, we introduce the auxiliary problem

$$
\begin{cases}-\operatorname{div}\left(h_{\varepsilon}^{3} P_{\varepsilon} \nabla w_{\varepsilon}\right)=-\operatorname{div}\left(\left|\nabla P_{\varepsilon}\right|^{q-2} \nabla P_{\varepsilon}\right) & \text { in } \Omega, \\ P_{0} h_{\varepsilon}^{3} \frac{\partial w_{\varepsilon}}{\partial \vec{n}}+a_{\varepsilon}(x, y) w_{\varepsilon}=\left|\nabla P_{\varepsilon}\right|^{q-2} \frac{\partial P_{\varepsilon}}{\partial n} & \text { on } \partial \Omega,\end{cases}
$$

where

$$
\begin{aligned}
& a_{\varepsilon}(x, y):=P_{0} G_{\varepsilon}^{-1}\left(h_{\varepsilon}^{3} \frac{\partial P_{\varepsilon}}{\partial n}-h_{\varepsilon}^{3} \frac{\partial G_{\varepsilon}}{\partial n}\right), \\
& G_{\varepsilon}(x):=\int_{0}^{x} \frac{6 \mu U_{0}}{h_{\varepsilon}^{2}(s)} \mathrm{d} s-k_{0}
\end{aligned}
$$

and

$$
k_{0}:=-\frac{\left(\left\|\partial P_{\varepsilon} / \partial n\right\|_{L^{\infty}(\partial \Omega)}^{q}+\left|U_{0}\right| h_{1}\right) C^{2}(\Omega) P_{0} h_{0}^{3}}{6 \mu} .
$$

Lemma 3.2. Problem (3.4) has a unique weak solution $w_{\varepsilon} \in V(\Omega)$.

Proof. As in Section 2 we consider the bilinear continuous and coercive form $A_{\varepsilon}$ : $V(\Omega) \times V(\Omega) \rightarrow \mathbb{R}$ defined by

$$
A_{\varepsilon}(\phi, \psi):=\int_{\Omega} h_{\varepsilon}^{3} P_{\varepsilon} \nabla \psi \cdot \nabla \phi \mathrm{d} x+\int_{\partial \Omega} a_{\varepsilon} \psi \phi \mathrm{d} \sigma .
$$

Since $P_{0} \leqslant P_{\varepsilon} \leqslant \sqrt{2 \bar{u}_{\varepsilon}}$, we obtain the continuity and coerciveness of $A_{\varepsilon}$ (as in Proposition 2.2). Applying the Lax-Milgram Theorem we conclude the proof.

Proof of Proposition 3.1. Taking $w_{\varepsilon}$ as test function in (3.3) we obtain

$$
\begin{aligned}
\int_{\Omega} P_{\varepsilon} h_{\varepsilon}^{3} \nabla P_{\varepsilon} \cdot \nabla w_{\varepsilon} \mathrm{d} \sigma= & \int_{\Omega} P_{\varepsilon} \nabla w_{\varepsilon} \cdot\left(6 \mu U h_{\varepsilon}, 0\right) \mathrm{d} \sigma \\
& +\int_{\partial \Omega} P_{0}\left(h_{\varepsilon}^{3} \frac{\partial P_{\varepsilon}}{\partial n}-\left\langle\left(6 \mu U h_{\varepsilon}, 0\right), n\right\rangle\right) w_{\varepsilon} \mathrm{d} \sigma_{2} .
\end{aligned}
$$

Taking $P_{\varepsilon}-P_{0}$ as test function in (3.4) it results

$$
\int_{\Omega} P_{\varepsilon} h_{\varepsilon}^{3} \nabla P_{\varepsilon} \cdot \nabla w_{\varepsilon} \mathrm{d} \sigma=\int_{\Omega}\left|\nabla P_{\varepsilon}\right|^{q} \mathrm{~d} \sigma
$$

Then we get

$$
\begin{aligned}
\int_{\Omega}\left|\nabla P_{\varepsilon}\right|^{q} \mathrm{~d} \sigma= & \int_{\Omega} P_{\varepsilon} 6 \mu U h_{\varepsilon} \frac{\partial w_{\varepsilon}}{\partial x} \mathrm{~d} \sigma \\
& +\int_{\partial \Omega} P_{0}\left(h_{\varepsilon}^{3} \frac{\partial P_{\varepsilon}}{\partial n}-\left\langle\left(6 \mu U h_{\varepsilon}, 0\right), n\right\rangle\right) w_{\varepsilon} \mathrm{d} \sigma_{2}
\end{aligned}
$$


Since $\left(6 \mu U h_{\varepsilon}, 0\right)=h_{\varepsilon}^{3} \nabla G_{\varepsilon}$, it results

$$
\int_{\Omega} P_{\varepsilon} 6 \mu U h_{\varepsilon} \frac{\partial w_{\varepsilon}}{\partial x} \mathrm{~d} \sigma=\int_{\Omega} P_{\varepsilon} h_{\varepsilon}^{3} \nabla G_{\varepsilon} \cdot \nabla w_{\varepsilon} \mathrm{d} \sigma
$$

Taking $G_{\varepsilon}$ as test function in (3.4) and using (3.6) we obtain

$$
\begin{aligned}
\int_{\Omega}\left|\nabla P_{\varepsilon}\right|^{q} \mathrm{~d} \sigma= & \int_{\Omega}\left|\nabla P_{\varepsilon}\right|^{q-2} \nabla P_{\varepsilon} \cdot \nabla G_{\varepsilon} \mathrm{d} \sigma \\
& -\int_{\partial \Omega} G_{\varepsilon}\left|\nabla P_{\varepsilon}\right|^{q-2} \frac{\partial P_{\varepsilon}}{\partial n} \mathrm{~d} \sigma_{2}+\left\langle G_{\varepsilon}, P_{0} h_{\varepsilon}^{3} \frac{\partial w_{\varepsilon}}{\partial n}\right\rangle_{H^{1 / 2}, H^{-1 / 2}} \\
= & \int_{\partial \Omega} P_{0}\left(h_{\varepsilon}^{3} \frac{\partial P_{\varepsilon}}{\partial n}-\left\langle\left(6 \mu U h_{\varepsilon}, 0\right), n\right\rangle\right) w_{\varepsilon} \mathrm{d} \sigma_{2} .
\end{aligned}
$$

Using the definition of $w_{\varepsilon}$ it results

$$
\int_{\Omega}\left|\nabla P_{\varepsilon}\right|^{q} \mathrm{~d} \sigma=\int_{\Omega}\left|\nabla P_{\varepsilon}\right|^{q-2} \nabla P_{\varepsilon} \cdot \nabla G_{\varepsilon} \mathrm{d} \sigma
$$

and then

$$
\int_{\Omega}\left|\nabla P_{\varepsilon}\right|^{q} \mathrm{~d} x \leqslant\left\|\nabla G_{\varepsilon}\right\|_{\left(L^{\infty}(\Omega)\right)^{2}} \int_{\Omega}\left|\nabla P_{\varepsilon}\right|^{q-1} \mathrm{~d} \sigma
$$

Consequently,

$$
\left\|\nabla P_{\varepsilon}\right\|_{\left(L^{q}(\Omega)\right)^{2}}^{q} \leqslant \frac{6 \mu U}{h_{0}^{2}}\left\|\nabla P_{\varepsilon}\right\|_{\left(L^{q-1}(\Omega)\right)^{2}}^{q-1} \leqslant C(\Omega) \frac{6 \mu U}{h_{0}^{2}}\left\|\nabla P_{\varepsilon}\right\|_{\left(L^{q}(\Omega)\right)^{2}}^{q-1},
$$

i.e. $\left\|\nabla P_{\varepsilon}\right\|_{\left(L^{q}(\Omega)\right)^{2}} \leqslant C(\Omega) 6 \mu U / h_{0}^{2}$, and since $P_{\varepsilon}=P_{0}$ on $\partial \Omega$, we obtain the desired result.

Proof of Theorem 1.2. Since $\left\|P_{\varepsilon}\right\|_{W^{1, q(\Omega)}} \leqslant K$, then there exists a subsequence $P_{i \varepsilon}$ which converges to $v$ weakly in $W^{1, q}(\Omega)$. Since $W^{1, q}(\Omega) \subset L^{\infty}(\Omega)$ is a compact embedding, it results $P_{\varepsilon} \rightarrow v$ in $L^{\infty}(\Omega)$. Taking limits in the weak formulation of (3.3) we obtain

$$
\int_{\Omega} v h^{3} \nabla v \cdot \nabla \psi \mathrm{d} x=\int_{\Omega} v \nabla \psi \cdot(6 \mu U h, 0) \mathrm{d} x \quad \forall \psi \in H_{0}^{1}(\Omega),
$$

which implies that $v$ is a weak solution to (1.2). Since $v \in W^{1, q}(\Omega)$ for any $1 \leqslant q<\infty$ and

$$
\|v\|_{W^{1, q(\Omega)}} \leqslant C\left(\Omega, h_{0}, P_{0}, \mu, U\right)
$$

taking limits as $q$ goes to $\infty$ we obtain $v \in W^{1, \infty}(\Omega)$. 


\section{Acknowledgements}

The author thanks J.I. Díaz for many helpful suggestions. The author is partially supported by DGES project REN 2000/0766 (Spain) and RTN HPRN-CT-2002-00274.

\section{References}

[1] G. Bayada, Inequations variationelles elliptiques avec conditions aux limites periodiques: application a la resolution de l'equation de Reynolds, These de 3eme cycle. Univ. de Lyon, 1972.

[2] B. Bhushan, Tribology and Mechanics of Magnetic Storage Devices, Springer, New York, 1990.

[3] A. Burgdorfer, The influence of the molecular mean free path on the performance of hydrodynamics gas lubricated bearings, J. Basic Eng. Trans. ASME 81 (1956) 94-100.

[4] A. Cameron, Principles of Lubrication, Longman, London, 1966.

[5] M. Chipot, On the Reynolds lubrication equation, Nonlinear Anal. Theory Meth. Appl. 12 (1988) $699-718$.

[6] M. Chipot, M. Luskin, Existence and uniqueness of solutions to the compressible Reynolds lubrication equation, SIAM J. Math. Anal. 17 (1986) 1390-1399.

[7] J.I. Díaz, J.I. Tello, On a problem lacking a classical solution in lubrication theory, Actas del XV-CEDYA Vol II, Vigo (1997) 429-434.

[8] H.G. Elrod, A derivation of the basic equations for hydrodynamic lubrication with a fluid having constant properties, Quart. Appl. Math. 17 (1959) 349-359.

[9] A. Friedman, J.I. Tello, Head-media interaction in magnetic recording, J. Differential Equations 171 (2001) 443-461.

[10] L. Rayleigh, Notes on the theory of lubrication, Philos. Mag. J. (1918) 1-12.

[11] O. Reynolds, On the theory of lubrication and its application to Mr Beauchamp Tower's experiments including an experimental determination of the viscosity of olive oil, Philos. Trans. R. Soc. 177 (1886) $157-234$.

[12] M. Rodríguez, A. Liñán, Cavitation in short bearings, AMSE J. Tribol. 107 (1985) 142-144.

[13] G.M. Troianiello, Elliptic Differential Equations and Obstacle Problems, Plenum Press, London, 1987. 